Research Article

\title{
Deep Learning-Based Positron Emission Tomography Molecular Imaging in the Assessment of Cognitive Dysfunction in Patients with Epilepsy
}

\author{
Mayila Tuerxun $\mathbb{D}^{1},{ }^{1}$ Lixin Yin $\mathbb{D}^{1},{ }^{1}$ Huiqun Chen $\mathbb{D}^{2},{ }^{2}$ and Jingqian Lin $\mathbb{D}^{3}$ \\ ${ }^{1}$ Department of Neurology, The First Affiliated Hospital of Xinjiang Medical University, Urumqi 830000, Xinjiang, China \\ ${ }^{2}$ Digestive Medicine, The $967^{\text {th }}$ Hospital of Joint Logistics Support Force of Chinese People's Liberation Army, Dalian 116000, \\ Liaoning, China \\ ${ }^{3}$ Department of Neurology, Yantai Laiyang Central Hospital, Laiyang 265200, Shandong, China
}

Correspondence should be addressed to Jingqian Lin; 150511133@stu.sxit.edu.cn

Received 14 July 2021; Revised 12 September 2021; Accepted 14 September 2021; Published 6 October 2021

Academic Editor: Gustavo Ramirez

Copyright (c) 2021 Mayila Tuerxun et al. This is an open access article distributed under the Creative Commons Attribution License, which permits unrestricted use, distribution, and reproduction in any medium, provided the original work is properly cited.

\begin{abstract}
This work aimed to investigate the application of positron emission tomography (PET) molecular imaging based on the deep learning algorithm in the assessment of cognitive dysfunction in patients with epilepsy. In this study, 52 hospitalized patients with epilepsy were selected as the epilepsy group and treated with different kinds of antiepileptic drugs, and 52 volunteers were selected as the control group. A U-net optimized network structure algorithm based on deep learning was proposed in this study and compared with a fully convolutional neural network (FCNN). Besides, it was applied in the PET molecular imaging of patients with epilepsy, and the segmentation effect of the U-net optimized network structure was good. According to event-related potential examinations, the proportion of patients with cognitive dysfunction in the epilepsy group (74.19\%) was higher than the proportion of the control group $(7.46 \%)(P<0.05)$. The patients with cognitive dysfunction $(57.89 \%)$ who took one antiepileptic drug were lower than those with two antiepileptic drugs $(84.61 \%)(P<0.05)$. The difference was statistically obvious in the overall quality of life of patients with epilepsy $(P<0.05)$. The occurrence of cognitive dysfunction in patients with epilepsy was related to the type of seizures. In addition, the quality of life of patients who suffered from cognitive dysfunction was low.
\end{abstract}

\section{Introduction}

Epilepsy is a group of chronic diseases and syndromes of central nervous system dysfunction caused by abnormal excessive discharge of brain neurons [1]. Epilepsy occurs in people of any age, region, and ethnicity. In recent years, the incidence of cerebrovascular disease, dementia, and neurodegenerative diseases has increased with the aging of the Chinese population. Moreover, the incidence of epilepsy in the elderly shows an upward trend [2]. According to the location of abnormal discharge of neurons and the range of discharge, the main clinical manifestations are the different dysfunctions, including different motor, sensory, cognitive, and autonomic nerve [3]. According to epidemiological research data, the annual prevalence of epilepsy is about
$0.8-4.5 \%$, and there are about 500,000 new epilepsy patients each year [4]. With the continuous updating of electronic medical equipment, humans' understanding of epilepsy has gradually deepened. The cognitive dysfunction caused by epilepsy has a major impact on the health and quality of life of patients, and long-term application of antiepileptic drugs brings patients' families severe economic pressure. Therefore, it has attracted the attention of many scholars [5]. Cognitive function means that the information is simply processed and transformed into its own psychological changes when the brain receives information from the outside world. In the process of receiving information, it mainly includes language, vision, hearing, comprehension, memory, and other aspects. Cognitive dysfunction refers to the abnormality of one or more of the above functions [6]. 
Kim et al. [7] pointed out that the intelligence score was generally lower than 75 points after the onset of epilepsy. Research data shows that about $35 \%$ of epilepsy patients will be accompanied by cognitive dysfunction, and patients will show obvious defects at the onset.

PET is considered to be one of the most promising imaging technologies. It can be applied to observe the relationship between brain functional activities and changes in blood flow metabolism in a living body. It is called "physiological tomography" and has been widely applied in the positioning of preoperative epilepsy lesions in recent years [8]. Deep learning is a type of machine learning algorithm that uses multiple layers to gradually extract higher-level features from the original input. In image processing, the lower layer can identify the edges, and the higher layer can identify the parts that are meaningful to humans [9]. Deep learning has achieved very marked effects in the computer vision. Besides, the U-net network structure is a biomedical image segmentation method obtained by further optimizing the full convolutional network algorithm. This network structure is divided into an upsampling path and a downsampling path and distributed in a symmetrical " $U$ " shape, so it was named "U-net" [10].

The U-net optimized network structure algorithm was proposed based on deep learning, compared with the fully convolutional neural network (FCNN) structure, and applied to the PET molecular images of 52 patients with epilepsy. The objective of this study was to explore the PET molecular imaging to assess the influencing factors of cognitive dysfunction in patients with epilepsy and the quality of life of patients with epilepsy.

\section{Materials and Methods}

2.1. Selection of Research Objects. In this study, 52 patients with epilepsy who were treated in hospital from November 17, 2017, to May 19, 2019, were selected as the epilepsy group. Besides, 52 healthy volunteers were taken as the control group. There were 53 males and 51 females, with an average age of $31.32 \pm 11.87$ years. This experiment had been authorized by the Medical Ethics Committee of Hospital, and the patients and their family members had understood the situation of this experiment and signed the informed consent forms.

The criteria for inclusion were defined to include patients who met the clinical and pathological diagnostic criteria of the International Antiepilepsy League's classifications of epilepsy and epilepsy syndrome and were 18-75 years old.

The criteria for exclusion were defined to include patients who suffered from cognitive dysfunction before the onset of epilepsy, had other cognitive dysfunctions, had mental retardation and were unable to cooperate with the examination, and suffered from major mental illnesses.

2.2. Treatment, Observation, and Classification of Patients with Epilepsy. The epilepsy group was treated with carbamazepine combined with butylphthalide soft capsules; $0.2 \mathrm{~g}$ butylphthalide soft capsules were taken three times a day, and $0.1 \mathrm{~g}$ carbamazepine tablet was also taken three times a day. The drug dose was adjusted reasonably according to the condition of epilepsy patients, and the highest dose was $0.3 \mathrm{~g}$. The treatment lasted for 6 months, and the outcome indicators were observed.

Before surgery, each patient should be asked about the cause of disease, age at first onset, clinical manifestations, time of onset, frequency of onset, past history (birth status, history of brain surgery, stroke, and brain nerve tissue disease), the administration of antiepileptic drugs (including the type of drugs, administration time, and dosage taken), family history of epilepsy, craniocerebral PET examination, and the assessment results of Quality of Life in Epilepsy-31 (QOLIE-31). According to the PET scanning and the number and type of epilepsies a year ago, the patients were classified as mild, moderate, and severe based on their specific conditions. The mild symptom meant that patients had no seizures within one year, and the moderate symptom referred to patients who were between mild and severe. What is more, the severe symptom was that patients suffered from more than 15 partial episodes within a year, more than 3 complex seizures, and more than 1 tonic spasm.

\subsection{Event-Related Potential Measurement. The Viking Quest} (produced by Nicol, USA) evoked potential meter was adopted, and the electrodes were placed in accordance with the international system method. The electrodes were placed at three positions (Ez, Dz, and $\mathrm{Qz})$. The reference electrode was set at the base of the nose, and the ground wire was placed at the right earlobe. The skin impedance was less than 5 gigabytes, and three sets of data were recorded at the same time. During the examination, the patient should maintain a quiet and relaxed mood and sit on the examination chair. Each patient needed to conduct a pretest in advance and understood the purpose and requirements of this experiment, and the short-, medium-, and high-frequency tones were recorded. The test was started after the patient could distinguish clearly. The target stimulation frequency was $3 \mathrm{KHz}$, the stimulation intensity was $85 \mathrm{~dB}$, and the random probability was set to $25 \%$. The nontarget stimulation frequency was $1 \mathrm{KHz}$, the intensity was $85 \mathrm{~dB}$, and the random probability was set to $75 \%$. Furthermore, the target stimulus was superimposed 450 times, the sensitivity was $10 \mu \mathrm{v}$, the bandpass range was set to $2-50 \mathrm{~Hz}$, and the analysis time was 1 second.

2.4. Assessment of Quality of Life in Patients with Epilepsy. The patients were assessed by the Chinese translation version of QOLIE-31 version 1.0. The total score of each question was divided by the corresponding number of questions, which was recorded as the initial score of each item. The product of the initial score of each item in the item table and the sum of the weight scores was taken as the total score. The higher the score was, the higher the patient's quality of life was. The cognitive function included 6 items that had the serial numbers $12,17,18,21,25$, and 29 in the scale, and the 
social functions contained 5 items that had the serial numbers $11,13,15,19$, and 22 in the scale.

2.5. PET Scanning of Patients with Epilepsy. The Siemens $\mathrm{PET} / \mathrm{magnetic}$ resonance (MR) all-in-one machine was used for imaging data collection. Before the scanning, all patients fasted for 6-8 hours, and their blood glucose levels were controlled at $3.8-6.2 \mathrm{mmol} / \mathrm{L}$. $\beta-2-\left[{ }^{18} \mathrm{~F}\right]-\mathrm{Flu}-$ oro-2-deoxy-D-glucose $\left({ }^{18} \mathrm{~F}\right.$-FDG) was intravenously injected with a dose of $0.2 \mathrm{mCi} / \mathrm{kg}$. The patient rested for 30 minutes in a calm environment and then was examined by the cranial PET/MR. Before the PET scanning, MR imaging (MRI) equipment was adopted to scan and locate the examined brain area. During the examination, the examination procedure should be explained to the patient. The patient was lying on the scanning table in a supine position and the head was fixed. The data collection time was set to 8 minutes, and the data was collected in 3D mode. During the scanning process, the physician needed to pay attention to the vital characteristics of the patient at any time.

2.6. U-Net Optimized Network Structure Algorithm. On the basis of the structure of U-net itself, data amplification methods were added so that overfitting would not occur when the data was small. U-net optimized network structure could be added to the cross-entropy loss function for two classification problems. The cross-entropy loss function (CLF) was calculated as follows:

$$
\operatorname{CLF}(q, y)=-\frac{1}{m} \sum_{i=1}^{m}\left[y_{i} \log \left(q_{i}\right)+\left(1-y_{i}\right)\right],
$$

or

$$
\operatorname{CLF}(q, y)= \begin{cases}-\log (q) & \text { if } y=1, \\ -\log (1-q), & \text { else. }\end{cases}
$$

In equations (1) and (2), $q$ represented the estimated value, and its value range was $[0,1]$. The value of $y$ was 0 or 1 , and $q$ was substituted by $q_{s}$, as shown in equation (3). Besides, equation (2) was transformed into equation (4).

$$
\begin{aligned}
q_{s} & = \begin{cases}q & \text { if } y=1, \\
1-q, & \text { else. }\end{cases} \\
\operatorname{CLF}(q, y) & =\operatorname{CLF}\left(q_{s}\right) \\
& =-\log \left(q_{s}\right) .
\end{aligned}
$$

The coefficient $\lambda_{s}$ was added on the basis of the crossentropy loss function to get equations (5) and (6).

$$
\begin{aligned}
\operatorname{CLF}\left(q_{s}\right) & =-\lambda_{s} \log \left(q_{s}\right) . \\
\lambda_{s} & = \begin{cases}b & \text { if } y=1, \\
1-b, & \text { else. }\end{cases}
\end{aligned}
$$

According to the above equations, the positive and negative of the sample were adjusted by adjusting the size of the value $b$, thereby affecting the total loss function. In general, the range of value $b$ was [0.5-1] to increase the weight of the positive sample loss function.

2.7. Evaluation Indexes of Segmentation Results. 3 indexes were applied in this study to evaluate the segmentation effect, namely, Dice similarity coefficient (DSC), sensitivity (SEN), and positive predictive value (PPV). DSC was a comprehensive index, which was often adopted to determine the coincidence between automatic segmentation results and actual values. Thus, DSC could be calculated as follows:

$$
\mathrm{DSC}=\frac{2 \mathrm{TPV}}{\mathrm{FPV}+\mathrm{FNV}+2 \mathrm{TPV}} \text {. }
$$

In equation (7), TPV stood for the number of true positive pixels, FPV represented the number of false positive pixels, and FNV expressed the number of false negative pixels. PPV was employed to calculate the ratio of the number of true positive pixels to the number of false positive pixels, and it could be expressed in the following equation:

$$
\mathrm{PPV}=\frac{\mathrm{TPV}}{\mathrm{TPV}+\mathrm{FPV}} .
$$

SEN was applied to calculate the ratio of the number of true positive pixels to the number of false negative pixels, which could be expressed as follows:

$$
\mathrm{SEN}=\frac{\mathrm{TPV}}{\mathrm{TPV}+\mathrm{FNV}} .
$$

The range of the above three evaluation indexes was $[0,1]$. The closer the calculation result was to 1 , the better the segmentation effect was; the closer the calculation result was to 0 , the worse the segmentation effect was.

2.8. Statistical Methods. In this study, SPSS 20.0 statistical analysis software was used for data processing. The standard deviation \pm variance was used for normal distribution; the average age of epidemiology, the quality of life score of patients, and the calculation data were expressed by percentage (\%). The DSC, SEN, and PPV of the two algorithms were compared, as well as the seizure type proportion and seizure frequency cases. In addition, $P<0.05$ meant that the difference was statistically substantial.

\section{Results}

3.1. Comparison on the Segmentation Effect of the Two Algorithms in the Reconstruction Model. The U-net optimized network structure algorithm was proposed based on deep learning and compared with the FCNN structure. Moreover, it was applied in the PET molecular images of 52 patients with epilepsy. The U-net optimized network structure contained 4 densely connected blocks in the upsampling and downsampling paths, and there was a densely connected block between the two. The number of densely connected blocks was different from the incremental rate. If the number of layers was denser inside the densely connected blocks, there were more parameters to be tested for the entire 
network structure. The DSC (89.47\%) of the U-net optimized network structure was higher than the DSC (84.56\%) of the FCNN structure, indicating that the two image segmentation methods had a higher degree of overlap between the segmentation results of this research algorithm and the actual value. The PPV of the U-net optimized network structure $(88.97 \%)$ was higher than the PPV of the FCNN structure $(85.15 \%)$, and the number of true positive pixels optimized by U-net was more. What is more, the SEN of U-net optimized network structure (89.51\%) was higher than the SEN (82.79\%) of the FCNN structure, and the difference was statistically obvious $(P<0.05)$ (Figure 1 ). Therefore, the U-net optimized network structure was better than the segmentation effect of the FCNN.

3.2. Epidemiological Data Statistics. There were 52 epilepsy patients included in this study, aged between 18 and 50 years, with an average age of $31.97 \pm 13.47$ years, including 28 males and 24 females. According to educational levels, 15 patients were undergraduate and above, and 37 cases were under high school. There were 52 patients $(25$ males and 27 females) in the control group, and they were 17-52 years old, with an average age of $32.16 \pm 12.04$ years. According to education levels, 18 patients were undergraduate and above, and 34 were under high school. The above differences were not statistically marked $(P>0.05)$ (Figures 2 and 3$)$.

3.3. Characteristics of Clinical Seizures in Patients with Epilepsy. In the 52 patients with epilepsy, 12 had partial seizures, which specifically included simple partial seizures, complex partial seizures, and partial seizures extending to generalized seizures; 24 patients had generalized seizures, including general absence seizures, generalized tonic spasm, and generalized myoclonus; and 16 patients had partial and generalized seizures (Figure 4). In the enrolled patients, 42 patients had monthly seizures less than or equal to 1 within one year, accounting for $80.76 \%$, and 10 patients had monthly seizures greater than 1 , accounting for $19.24 \%$. The difference between the two was statistically substantial $(P<0.05)$ (Figure 5).

3.4. PET/MRI Molecular Imaging Characteristics of Patients with Epilepsy. The tuberous sclerosis 2 (TSC2) gene was mutated at c.600-2 A $>$ G. The images of brain MRI showed that cortical or subcortical nodules presented the high signals on T2-weighted imaging (T2WI), T2 FLAIR, and double inversion recovery sequence (DIR), as well as the low signals on T1WI sequence. $\quad{ }^{18} \mathrm{~F}$-fluorodeoxyglucose $\quad\left({ }^{18} \mathrm{~F}-\mathrm{FDG}\right) \quad$ PET/MRI indicated that the aforementioned abnormal signal areas had low-metabolism areas matching the size of the nodules themselves. At the left anterior temporal area where the scalp EEG indicated the epileptic area, PET/MRI showed that there was extensive low metabolism, and the metabolic area was dramatically larger than the nodule itself. For the nonepileptogenic nodules, the left posterior temporal area and the left occipital area of the nodule had the same low-metabolic range as the nodule itself. The above results suggested that

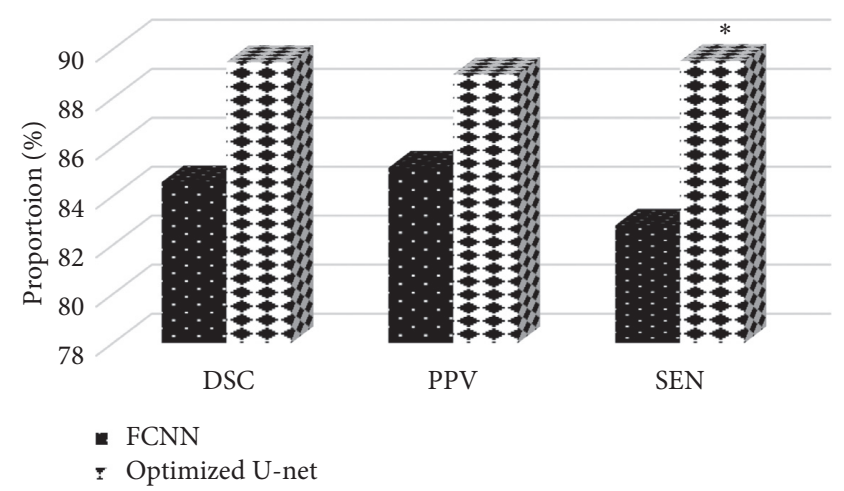

Figure 1: Comparison on the segmentation effects of the two algorithms in the reconstruction model.

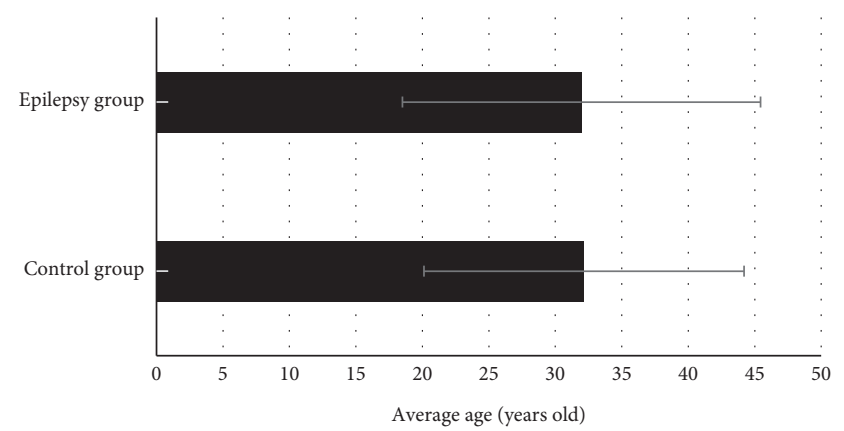

Figure 2: Comparison on the average age of subjects from the control group and the epilepsy group.

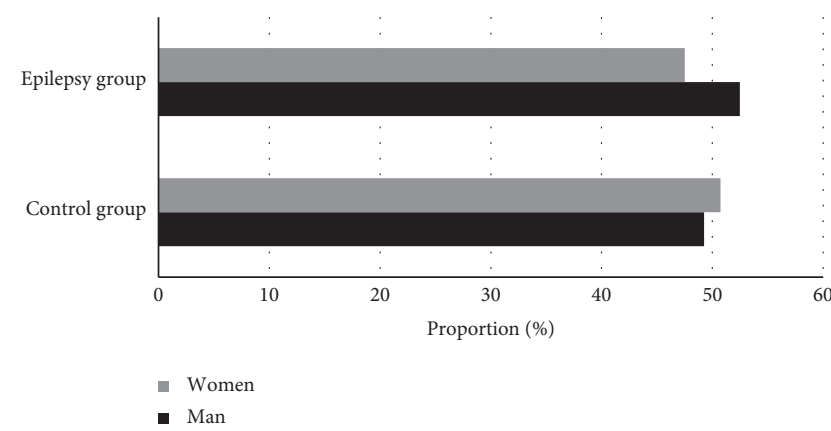

FIGURE 3: Comparison on the gender of subjects from the control group and the epilepsy group.

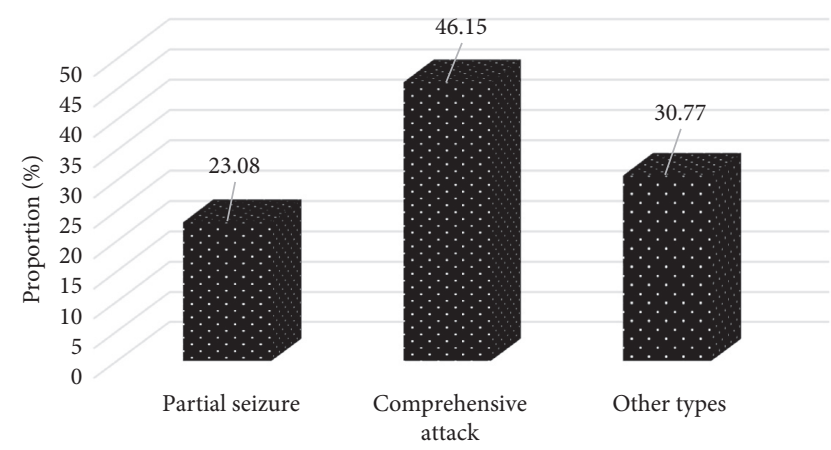

FIgURE 4: Types of seizures in patients with epilepsy. 


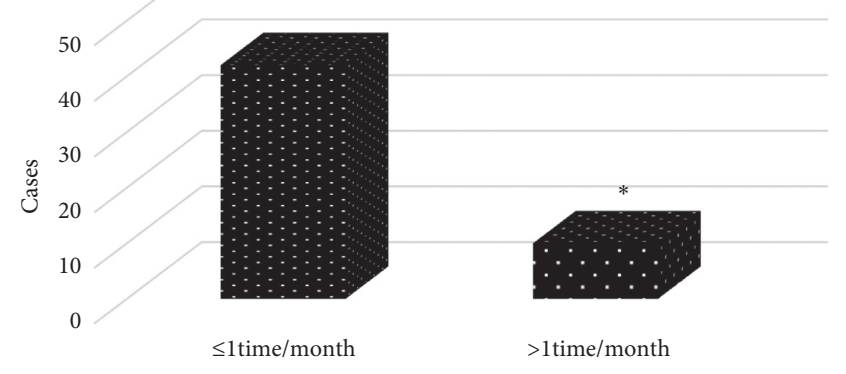

Figure 5: Comparison on frequency of epilepsy patients (note: * indicated that the difference was statistically obvious in contrast to less than or equal to 1 time/month $(P<0.05))$.

epileptogenic nodules could be combined with abnormal lowmetabolic cortex. The aforementioned imaging features were helpful to identify epileptogenic nodules. There was excessive discharge of the lesion, rapid and repeated depolarization of a large number of neuron cell membranes, and a significant increase in energy consumption, resulting in an obvious growth of local blood flow and glucose metabolism; ${ }^{18} \mathrm{~F}$-FDG PET imaging showed a high metabolic focus (Figures 6 and 7).

3.5. PET Examination Results of Patients with Epilepsy. In this study, 52 patients with epilepsy completed the PET molecular imaging examinations, and it was found that there were no obvious abnormal changes in the brains of 12 patients. Obvious lesions were found in 40 patients, accounting for $76.9 \%$. Among them, there were 10 patients with hippocampal lesions (19.23\%), 16 patients with frontal lobe lesions (30.77\%), and 8 patients with temporal lobe lesions (15.38\%). There were 6 cases of parietal disease, accounting for $11.54 \%$ (Figure 8 ).

3.6. Results of Event-Related Potentials in Patients with Epilepsy. The 52 epilepsy patients enrolled in the group completed the event-related potential examinations, showing that there were 14 patients with normal cognitive function (25.81\%). However, there were 48 patients with normal cognitive function in the control group, accounting for $92.54 \%$. The difference between the two was statistically great $(P<0.05)$. There were 38 patients with cognitive dysfunction in the epilepsy group, occupying $74.19 \%$, and 4 patients in the control group with cognitive dysfunction, accounting for $7.46 \%$. Thus, the difference was statistically substantial in the number of cases with cognitive dysfunction from the two groups $(P<0.05)$ (Figure 9$)$.

3.7. Influence of Education Levels of Epilepsy Patients on Cognitive Function. In the 52 patients with epilepsy, the highest degree was postgraduate, and the lowest degree was primary school. Based on the degree of education, there were 15 patients with bachelor degree or above and 37 patients with high school degree or below. Moreover, 11 patients with normal cognitive function and 19 patients with cognitive dysfunction had the bachelor degrees or above. There

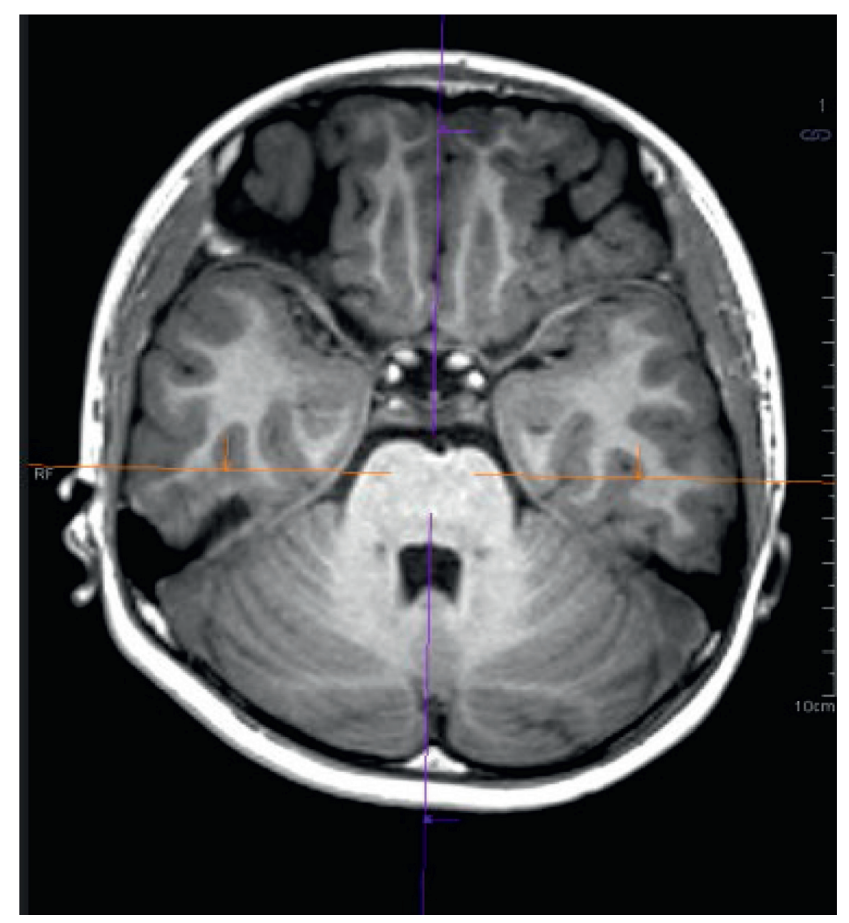

Figure 6: CT image of polyps (note: the image showed that the polyps protruded to the inside of the intestinal cavity, which was in a large amount and dense).

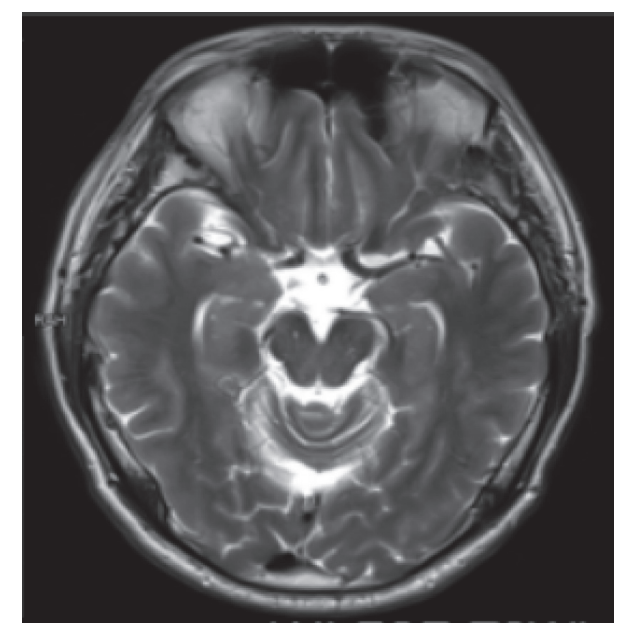

FIgURE 7: An image of polyp cancer (note: the local tube wall was thickened, with irregular edges).

were 8 patients with normal cognitive function and 14 patients with cognitive dysfunction who were with high school degrees or below. The above differences were not statistically obvious $(P>0.05)$ (Figure 10$)$.

3.8. Medications and Cognitive Function of Epilepsy Patients. In the 52 patients, 19 patients took a kind of antiepileptic drug, among which 11 cases (57.89\%) developed cognitive dysfunction. There were 26 patients taking two or more antiepileptic drugs, among which $22(84.61 \%)$ had cognitive dysfunction. 7 patients were not given antiepileptic drugs, 


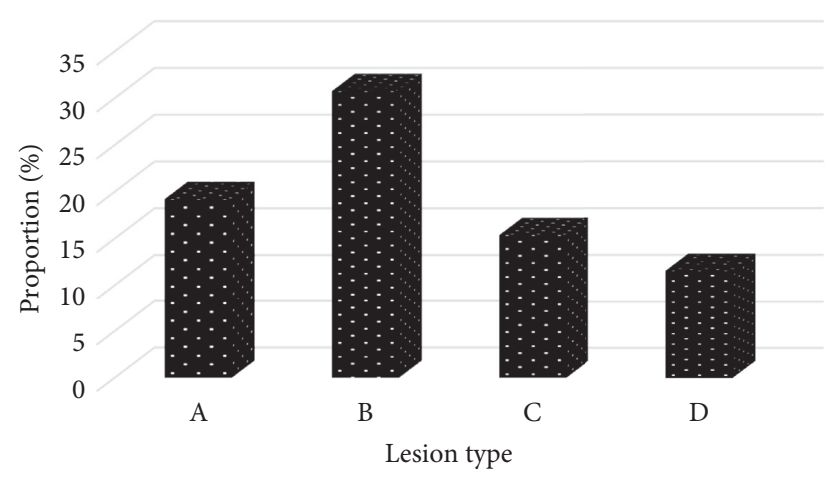

FIgUre 8: Brain PET examination results of patients with epilepsy (note: A, B, C, and D stood for the hippocampal lesion, frontal lobe lesion, temporal lobe lesion, and parietal lobe lesion, respectively).

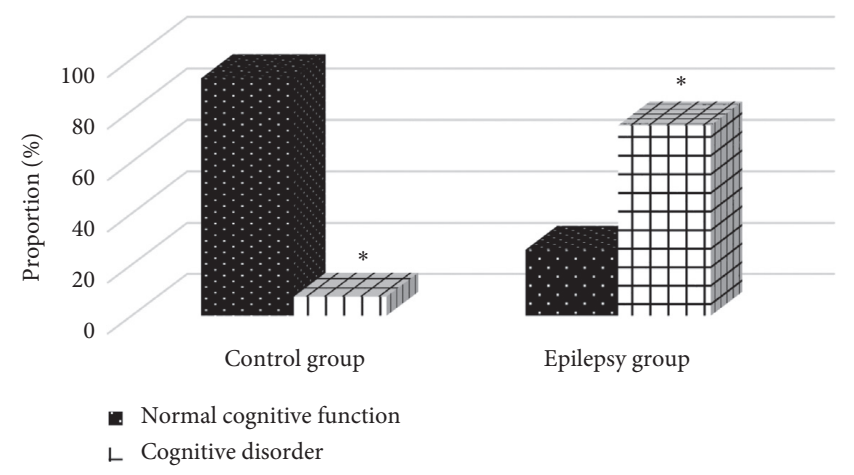

FIGURE 9: Results of event-related potentials in patients with epilepsy.

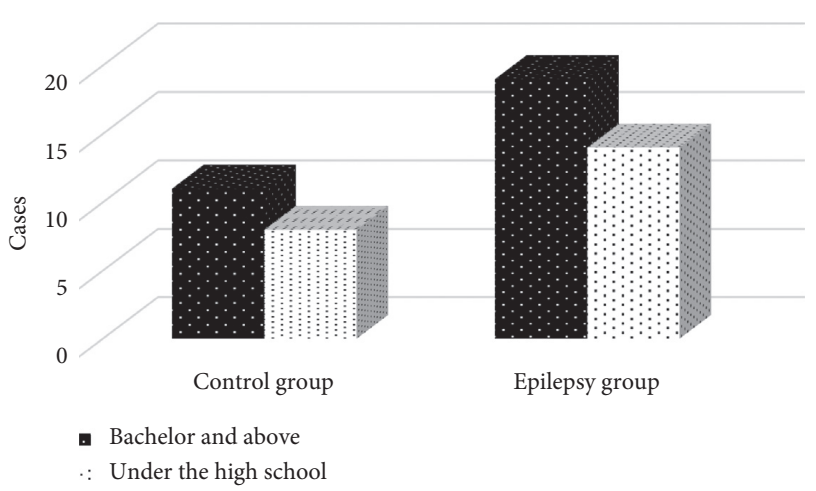

FIGURE 10: Influence of education levels on cognitive function in epilepsy patients.

and only 1 case had cognitive dysfunction. The difference between any two groups was statistically marked $(P<0.05)$ (Figure 11).

3.9. Quality of Life Scores of Patients with Epilepsy. The quality of life in 52 patients with epilepsy was scored by QOLIE-31, and the standard score method was adopted to improve the score and total score value of each project. The cognitive and social function scores in epilepsy group were significantly higher than those in control group $(3.12 \pm 0.34$

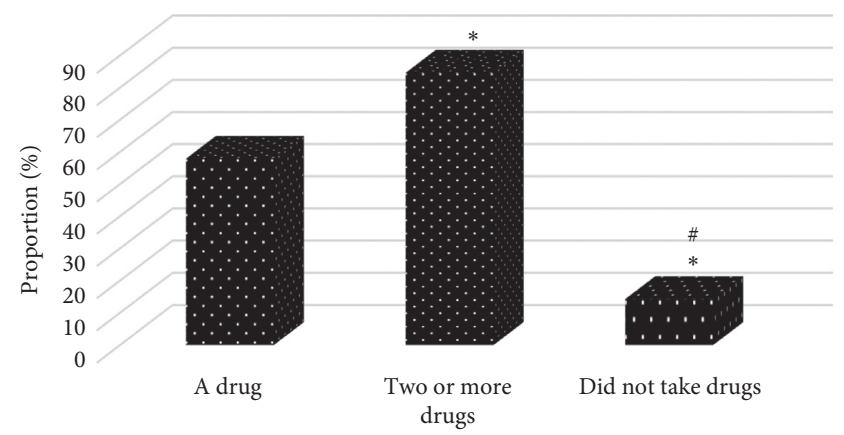

FIgURE 11: Influences of medication on cognitive function in patients with epilepsy (note: ${ }^{*}$ meant that the difference was statistically huge in contrast to taking one drug $(P<0.05) ;{ }^{*}$ indicated that there was a statistically remarkable difference in contrast to taking two or more drugs $(P<0.05))$.

versus $3.68 \pm 0.79 ; 3.56 \pm 0.23$ points versus $3.74 \pm 0.26$ points), and the difference was statistically significant $(P<0.05)$. It was found that the quality of life of patients with cognitive dysfunction was relatively poor (Figure 12).

\section{Discussion}

Epilepsy is a common neurological disease. More than $30 \%$ of patients with diagnosed epilepsy will have cognitive dysfunction [11]. In the past, people mainly paid attention to how to control epileptic seizures but ignored the cognitive dysfunction caused by epilepsy and the quality of life of patients with epilepsy. With the continuous development of medical high-tech equipment, people's attention to cognitive dysfunction caused by epilepsy has gradually increased [12]. Hasan et al. [13] explored a variety of factors that caused cognitive dysfunction in patients with epilepsy, including factors such as gender, age, seizure frequency, seizure type, and antiepileptic drug administration. They found that the age, frequency of seizures, type of seizures, and drug use were all related to cognitive dysfunction. In particular, the effect of frequency and type of seizures on cognitive function was very obvious, which was consistent with the results of this study. The event-related potential examinations were applied in this study, and the results suggested that seizure frequency, seizure type, brain PET molecular imaging, and drug use were all influencing factors of cognitive dysfunction, which were in line with the above research viewpoints. Chen et al. [14] retrospectively analyzed the cognitive dysfunction of 129 patients with epilepsy. The most serious damage to cognitive function was generalized tonic spasm, followed by complex partial seizures. If the frequency of complex partial seizures was more than 2 times/month, it would cause a serious decline in the patient's memory and mobility. Postulart et al. [15] believed that complex partial seizures and extension of partial seizures to generalized seizures were likely to cause damage to cognitive function. It indicated that cognitive dysfunction was highly correlated with the type of seizure, which was in accordance with the viewpoint of this study.

The most common way to treat epilepsy is to take antiepileptic drugs, which can reduce cognitive dysfunction 


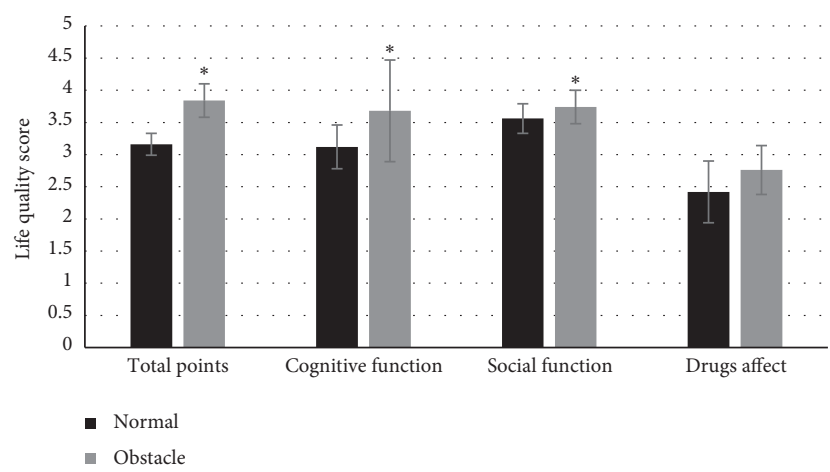

FIGURE 12: Quality of life score and cognitive function in patients with epilepsy (note: ${ }^{*}$ showed that the difference was statistically substantial compared with normal cognitive function $(P<0.05))$.

caused by epilepsy through antiepileptic drugs. However, antiepileptic drugs have side effects, and the dosage and frequency of administration will also have a certain impact on cognitive function [16]. Liguori et al. [17] pointed out that the more types of antiepileptic drugs were applied, the more obvious the degree of cognitive impairment in patients was. Oldan et al. [18] thought that antiepileptic drugs could inhibit the conduction of neuronal excitement and hinder the transmission of neurotransmitters, thereby affecting the cognitive function of patients. It was found that patients taking two or more antiepileptic drugs could promote the occurrence of cognitive dysfunction, indicating that the type of drug taken was one of the independent risk factors that affected cognitive dysfunction. The QOLIE-31 was adopted to evaluate the quality of life of patients with epilepsy, so that the results of this study had good credibility. Ogundare et al. [19] used QOLIE-31 to assess 85 patients with epilepsy and healthy controls and found that the scores of epilepsy patients were sharply lower than the scores of the control group. In addition, patients with cognitive dysfunction had the lowest scores, indicating that epilepsy-induced cognitive dysfunction could reduce the quality of life of patients. The results of this study revealed that the differences in the overall quality of life, cognitive function, and social function of patients with epilepsy were statistically obvious $(P<0.05)$, which was consistent with the results of the above research.

\section{Conclusion}

The U-net optimized network structure algorithm was proposed based on deep learning. Besides, it was compared with the FCNN structure and applied to the PET molecular images of 52 patients with epilepsy. U-net optimized network structure had better segmentation effect than FCNN. The occurrence of cognitive dysfunction in patients with epilepsy was related to the type of seizures, the frequency of seizures, the consumption of antiepileptic drugs, and brain PET molecular imaging. The total score of quality of life, cognitive function, and social function scores of patients with cognitive dysfunction were lower compared to patients with normal cognitive function, and their quality of life was low. The limitation of this study is that there are fewer case samples collected within a certain period of time, which may cause certain deviations in the results of this study. Later, it is considered to expand the sample range for further improvement. In short, the treatment of epilepsy cannot be through a single drug. It was also necessary to consider the adverse effects of cognitive dysfunction caused by epilepsy. The patients' attention to cognitive function has been increased to a certain extent, and their overall quality of life has been improved.

\section{Data Availability}

The data used to support the findings of this study are available from the corresponding author upon request.

\section{Conflicts of Interest}

The authors declare no conflicts of interest.

\section{References}

[1] V. K. Pilli, J. W. Jeong, P. Konka, A. Kumar, H. T. Chugani, and C. Juhász, "Objective PET study of glucose metabolism asymmetries in children with epilepsy: implications for normal brain development," Human Brain Mapping, vol. 40, no. 1, pp. 53-64, 2019.

[2] C. E. Popescu, R. Mai, R. Sara et al., "The role of FDG-PET in patients with epilepsy related to periventricular nodular heterotopias: diagnostic features and long-term outcome," Journal of Neuroimaging: Official Journal of the American Society of Neuroimaging, vol. 29, no. 4, pp. 512-520, 2019.

[3] S. Desarnaud, C. Mellerio, F. Semah et al., "18F-FDG PET in drug-resistant epilepsy due to focal cortical dysplasia type 2 : additional value of electroclinical data and coregistration with MRI," European Journal of Nuclear Medicine and Molecular Imaging, vol. 45, no. 8, pp. 1449-1460, 2018.

[4] S. Elwan, A. Alexopoulos, D. C. Silveira, and P. Kotagal, "Lateralizing and localizing value of seizure semiology: comparison with scalp EEG, MRI and PET in patients successfully treated with resective epilepsy surgery," Seizure, vol. 61, pp. 203-208, 2018.

[5] Y.-H. Wang, Y. An, X.-T. Fan et al., "Comparison between simultaneously acquired arterial spin labeling and 18F-FDG PET in mesial temporal lobe epilepsy assisted by a PET/MR system and SEEG," NeuroImage: Clinic, vol. 19, pp. 824-830, 2018.

[6] K. Shang, J. Wang, X. Fan et al., "Clinical value of hybrid TOFPET/MR imaging-based multiparametric imaging in localizing seizure focus in patients with MRI-negative temporal lobe epilepsy," American Journal of Neuroradiology, vol. 39, no. 10, pp. 1791-1798, 2018.

[7] J. A. Kim, J. W. Jeong, M. E. Behen et al., "Metabolic correlates of cognitive function in children with unilateral Sturge-Weber syndrome: evidence for regional functional reorganization and crowding," Human Brain Mapping, vol. 39, no. 4, pp. 1596-1606, 2018.

[8] X.-Q. Wu, Y.-N. Zhao, J. Ding et al., "Decreased vesicular acetylcholine transporter related to memory deficits in epilepsy: a [18 F] VAT positron emission tomography brain imaging study," Epilepsia, vol. 59, no. 9, pp. 1655-1666, 2018.

[9] M. Hu, Y. Zhong, S. Xie, and L. Haibin, L. Zhihan, "Fuzzy system based medical image processing for brain disease 
prediction," Frontiers in Neuroscience, vol. 15, Article ID 714318, 2021.

[10] Z. Yu, S. U. Amin, M. Alhussein, and L. Zhihan, "Research on disease prediction based on improved DeepFM and IoMT," IEEE Access, vol. 9, no. 99, p. 1, 2021.

[11] H. Seo, C. Huang, M. Bassenne, R. Xiao, and L. Xing, "Modified U-net (mU-Net) with incorporation of objectdependent high level features for improved liver and livertumor segmentation in ct images," IEEE Transactions on Medical Imaging, vol. 39, no. 5, pp. 1316-1325, 2020.

[12] Y. Li, J. Zhao, Z. Lv, and J. Li, "Medical image fusion method by deep learning," International Journal of Cognitive Computing in Engineering, vol. 2, pp. 21-29, 2021.

[13] S. M. Kamrul Hasan and C. A. Linte, "A modified U-Net convolutional network featuring a nearest-neighbor re-sampling-based elastic-transformation for brain tissue characterization and segmentation," in Proceedings of the 2018 IEEE Western New York Image and Signal Processing Workshop (WNYISPW), Rochester, NY, USA, October 2018.

[14] S. Chen, A. Qin, D. Zhou, and D. Yan, "Technical note: U-netgenerated synthetic CT images for magnetic resonance imaging-only prostate intensity-modulated radiation therapy treatment planning," Medical Physics, vol. 45, no. 12, pp. 5659-5665, 2018.

[15] D. M. IJff, D. Postulart, D. A. J. E. Lambrechts et al., "Cognitive and behavioral impact of the ketogenic diet in children and adolescents with refractory epilepsy: a randomized controlled trial," Epilepsy and Behavior, vol. 60, pp. 153-157, 2016.

[16] D. Chen, P. Wawrzynski, and Z. Lv, "Cyber security in smart cities: a review of deep learning-based applications and case studies," Sustainable Cities and Society, vol. 66, Article ID 102655, 2020.

[17] C. Liguori, C. Costa, F. Franchini et al., "Cognitive performances in patients affected by late-onset epilepsy with unknown etiology: a 12-month follow-up study," Epilepsy and Behavior: E \& B, vol. 101, Article ID 106592, 2019.

[18] J. D. Oldan, H. W. Shin, A. H. Khandani, C. Zamora, T. Benefield, and V. Jewells, "Subsequent experience in hybrid PET-MRI for evaluation of refractory focal onset epilepsy," Seizure, vol. 61, pp. 128-134, 2018.

[19] T. Ogundare, T. O. Adebowale, and O. A. Okonkwo, "Quality of life among patients with epilepsy in Nigeria: predictors and barriers to routine clinical use of QOLIE-31," Quality of Life Research, vol. 30, no. 2, pp. 487-496, 2020. 